PBAR Note 646

\title{
Measurements of Beam Momentum at the Stacking Energy of the Accumulator
}

\author{
Steve Werkema \\ November 29, 2000
}

\begin{abstract}
The momentum and orbit length of beam on the central and extraction orbits of the Antiproton Source Accumulator are calculated from measurements of revolution frequency and transverse beam position. This report gives the results of measurements made at the stacking energy before and after the 1998-99 Accumulator lattice upgrade.

Before the lattice upgrade, the Accumulator central orbit length and momentum are measured to be: $L_{c}=474.0433 \pm 0.0025 \mathrm{~m}, p_{c}=8819.5 \pm 4.1 \mathrm{MeV} / \mathrm{c}$. After the lattice upgrade the central orbit length and momentum are measured to be: $L_{c}=474.0532 \pm 0.0016 \mathrm{~m}, p_{c}=8803.4 \pm 2.7 \mathrm{MeV} / \mathrm{c}$.

The extraction orbit length and momentum prior to the lattice upgrade are measured to be: $L_{e}=474.1916 \pm 0.0054 \mathrm{~m}, p_{e}=8900.8 \pm 9.2 \mathrm{MeV} / \mathrm{c}$. After the lattice upgrade the extraction orbit length and momentum are measured to be: $L_{e}=474.1599 \pm 0.0036 \mathrm{~m}, p_{e}=8886.3 \pm 6.1 \mathrm{MeV} / \mathrm{c}$.
\end{abstract}

\section{Introduction}

One beneficial spin-off of operating the Antiproton Source for Fermilab experiment E835 is the ability to make calibrated measurements of the Accumulator beam energy ${ }^{1}$. This memo describes measurements of the Accumulator beam energy on the central and extraction orbits at its design energy. The analysis presented here includes measurements from before and after the Accumulator lattice upgrade. The results of this analysis are summarized in Table 2 on page 6.

The beam energy is determined by measuring the average velocity of the beam. The velocity of the beam is derived from measurements of the revolution frequency and the orbit length. These measurements are conducted with bunched beam; consequently, the revolution frequency is inferred from the frequency of the RF used to bunch the beam. The orbit length is calculated by comparing the horizontal closed orbit of the beam with a reference orbit of known length, $L_{r e f}$. An orbit measurement consists of the readout of all of the Accumulator beam position monitors (BPMs). The orbit length is calculated as a correction to the length of the reference orbit. To first order, the orbit length can be written as:

$$
L=L_{r e f}+\Delta L
$$

where $L_{r e f}$ is the length of the reference orbit. The first order correction, $\Delta L$, can be written as:

$$
\Delta L=\sum_{i=1}^{N_{b p m}} C_{i} \Delta x_{i}
$$

The sum in equation (2) runs over all operational horizontal BPMs. $\Delta L$ depends only on the difference between the measured orbit and the reference orbit at each horizontal BPM $\left(\Delta x_{i}\right)$. The constants, $C_{i}$, depend entirely on the Accumulator lattice.

\section{The Reference Orbit}

The reference orbit is an orbit measurement made when the beam energy is precisely known. If the beam energy is known, the length of the reference orbit is determined from the beam energy 
and the revolution frequency. The reference orbit used in the calculation of all of the beam energy measurements presented here was obtained from E835 data taken at the $\psi^{\prime}$ Charmonium resonance on June 14, 2000 The horizontal BPM readout for this orbit is given in Appendix 1.

The details of the orbit length calculation and the determination of a reference orbit are given in reference 1

\section{Estimating the Uncertainty in the Beam Energy Measurement}

The uncertainty in the measurement of the $8 \mathrm{GeV}^{3}$ central orbit energy of the Accumulator arises from the uncertainty in the length of the orbit and the uncertainty in the revolution frequency of the beam $(f)$. The overall variation in the beam momentum, in terms of these two contributions is given by:

$$
\frac{d p}{p}=\gamma^{2}\left(\frac{d f}{f}+\frac{d L}{L}\right)
$$

Since this technique of the beam energy determination is accomplished by a velocity measurement, one expects to pay a big price as the beam becomes relativistic. Equation (3) shows that the uncertainty in the momentum measurement grows as $\gamma^{3}$ when the beam energy is increased.

\section{Uncertainty in the measurement of the revolution frequency}

The frequency of the RF used to bunch the beam for closed orbit measurements can be determined to within approximately $0.1 \mathrm{~Hz}$ (at the revolution harmonic). Thus, if the RF frequency correctly gives the average revolution frequency of the beam "seen" by the BPMs, the $d f$ contribution to the beam momentum measurement uncertainty is very small. The $0.1 \mathrm{~Hz}$ uncertainty in $f$ gives rise to a $0.13 \mathrm{MeV} / \mathrm{c}$ contribution to the error in beam momentum at $8 \mathrm{GeV}$ on the central orbit. This small contribution to the uncertainty in the momentum measurement will be neglected in what follows.

\section{Uncertainty in the measurement of the orbit length}

Equation (3) indicates that, at beam kinetic energies near $8 \mathrm{GeV}$, each $1 \mathrm{~mm}$ uncertainty in the orbit length corresponds to a $1.66 \mathrm{MeV} / \mathrm{c}$ uncertainty in the beam momentum. There are a number of contributors to the uncertainty in the orbit length. These sources of error will be enumerated here.

\section{Uncertainty in the length of the reference orbit}

The calibration of the reference orbit is described in reference 1. The uncertainty in the length of the reference orbit, $\delta L_{r e f}$, is primarily due to the uncertainty in the mass of the $\psi^{\prime}$ resonance. The July 2000 PDG Particle Physics Booklet reports the uncertainty in the mass of the $\psi^{\prime}$ to be $0.09 \mathrm{MeV} / \mathrm{c}^{2}$. From this it is determined that $\delta L_{\text {ref }}=0.6 \mathrm{~mm}$.

\section{Neglect of second and higher order terms}

The neglected second order term in the orbit length calculation is derived in an appendix to reference 1 The second order term, $\delta L_{2}$, is given by:

$$
\delta L_{2}=\oint\left[x_{r}^{\prime} \Delta x^{\prime}+y_{r}^{\prime} \Delta y^{\prime}+\frac{1}{2}\left(\Delta x^{\prime 2}+\Delta y^{\prime 2}\right)\right] d s
$$

$x_{r}$ and $y_{r}$ are the horizontal and vertical displacements of the reference orbit from the central orbit of the Accumulator, and $\Delta x$ and $\Delta y$ are the horizontal and vertical displacements of the orbit 
being measured from the reference orbit. The integral of equation (4) follows the closed orbit for one circuit around the Accumulator.

$\delta L_{2}$ is a very small length. This can be seen by making the perverse assumptions that $x_{r}{ }^{\prime}, y_{r}{ }^{\prime}, \Delta x^{\prime}$, and $\Delta y^{\prime}$ are each $1 \mathrm{mrad}$ everywhere ${ }^{4}$, and that all of the terms in the integrand add coherently. Under these assumptions, $\delta L_{2} \sim 3 \times(1 \mathrm{mrad})^{2} \times 474.05 \mathrm{~m}=1.4 \mathrm{~mm}$. If these assumptions were actually realized, this term would contribute about $2.3 \mathrm{MeV} / \mathrm{c}$ to the uncertainty in the beam momentum. This is very likely a grossly overestimated upper bound to the actual contribution of this term.

\section{Lattice different from when the reference orbit was measured}

The orbit length calculation utilizes a model of the horizontal orbit that is parameterized as a superposition of dipole kicks from various elements of the Accumulator lattice. The coupling of these kicks to the closed orbit distortion depends on the lattice functions at the location of the kick and at the location of the orbit distortion. Kicks from only the horizontal dipole magnets are sufficient to parameterize the horizontal orbit when the lattice is not significantly different from what it was when the reference orbit was measured. However, if the lattice is appreciably different from what it was at the time the reference orbit was measured, kicks from the horizontal dipoles alone are not sufficient to accurately model the horizontal orbit. When the lattice differs from the reference orbit lattice (as is the case for $8 \mathrm{GeV}$ measurements) the orbit model is expanded to allow kicks from the Accumulator quadrupoles as well as the horizontal dipoles. Reference 1 describes how this is accomplished in the orbit length calculation as well as a test of this technique using orbits of known length that were measured before and after the Accumulator lattice upgrade. The conclusion of this test is that a change in the lattice from the reference orbit lattice has a very small impact on the calculated orbit length ${ }^{5}$.

\section{Uncertainties in the BPM readout}

Errors in the readout of each BPM will contribute an amount, $\delta L_{B P M}$, to the uncertainty in the orbit length measurement. Since only difference orbits are used, the orbit length calculation is insensitive to any offsets that in the BPM readout that are common to both the reference orbit and the measured orbit. Thus, only gain and scale errors in the BPM electronics and associated processing have an impact on the measurement of the orbit length. While an accurate assessment of these errors is not available, there is evidence that the BPMs are correctly scaled to within $15 \%$.

The impact of errors of scale in the BPM readout was obtained by a Monte Carlo error analysis. In this analysis, 5000 orbits are generated by multiplying the original readout of each BPM by randomly distributed scale factors. The scale factors were generated according to a Gaussian distribution with a mean of 1.0 and a $\sigma$ of 0.2 . This scale factor distribution represents a very conservative overestimate of the BPM scaling error. Figure 1 and Figure 2 show the resulting $\Delta L$ distributions for a post-lattice upgrade measurement and a pre-lattice upgrade measurement respectively. The width of resulting distribution of orbit lengths is an estimate of $\delta L_{B P M}$. The magnitude of $\delta L_{B P M}$ depends on the orbit. If the orbit differs significantly from the reference orbit (as is the case for the pre-upgrade measurements) the uncertainty will be large.

Figure 1 shows the worst case (i.e. the widest) $\Delta L$ distribution of all the post-upgrade measurements. The rms width of this distribution is $0.45 \mathrm{~mm}$. Therefore a conservative estimate for the post-upgrade BPM readout uncertainty is $\delta L_{B P M}=0.45 \mathrm{~mm}$. This value of $\delta L_{B P M}$ corresponds to a momentum error of $0.74 \mathrm{MeV} / \mathrm{c}$ at $8 \mathrm{GeV}$. Figure 2 shows the worst case $\Delta L$ distribution of all the pre-upgrade measurements. The rms width of this distribution is $1.96 \mathrm{~mm}$. 
The resulting estimate for the pre-upgrade BPM readout uncertainty is $\delta L_{B P M}=1.96 \mathrm{~mm}$. This value of $\delta L_{B P M}$ gives rise to a momentum error of $3.26 \mathrm{MeV} / \mathrm{c}$ at $8 \mathrm{GeV}$.



Figure 1 Histogram of the results of 5000 calculations of the first order correction, $\Delta L$, to the $6 / 14 / 2000 \psi^{\prime}$ reference orbit for the 3/12/2000 orbit measurement. Each entry is calculated from a the original horizontal BPM difference orbit multiplied by a scale factor that is randomly generated according to a gaussian distribution with a mean of 1.0 and a $\sigma$ of 0.2 .



Figure 2 Histogram of the results of 5000 calculations of the first order correction, $\Delta L$, to the $6 / 14 / 2000 \psi^{\prime}$ reference orbit for the 9/24/1996 orbit measurement. Each entry is calculated from a the original horizontal BPM difference orbit multiplied by a scale factor that is randomly generated according to a gaussian distribution with a mean of 1.0 and a $\sigma$ of 0.2 .

\section{Revolution frequency and Bend field not at nominal field}

The definition of the central orbit in the Accumulator requires the specification of two numbers the revolution frequency, $f_{c}$, and the magnetic field measured in the Accumulator reference dipole $B_{o}$. The values $f_{c}$ and $B_{o}$ before and after the Accumulator lattice upgrade are given in Table 1 In general, the revolution frequency and field are different from the canonical $f_{c}$ and $B_{o}$ 
resulting in a radial displacement of the beam from the central orbit. Consequently, a measurement of the central orbit energy requires a correction to account for the energy difference between a radially displaced orbit and the nominal central orbit.

Table 1: Nominal central and extraction orbit revolution frequencies $\left(f_{c}, f_{e}\right)$ and the reference field, $B_{o}$, before and after the Accumulator lattice upgrade

\begin{tabular}{|c||c|c|c|}
\hline & $\boldsymbol{f}_{\boldsymbol{c}}(\mathrm{Hz})$ & $\boldsymbol{f}_{\boldsymbol{e}}(\mathrm{Hz})$ & $\boldsymbol{B}_{\boldsymbol{o}}$ (Gauss) \\
\hline \hline Before Lattice Upgrade & 628867.0 & 628734.3 & 16742.0 \\
\hline After Lattice Upgrade & 628841.0 & 628765.0 & 16714.4 \\
\hline
\end{tabular}

If the revolution frequency difference, $\Delta f$, and field difference, $\Delta B$, are small, the momentum correction is given by:

$$
\frac{\Delta p}{p}=-\frac{1}{\eta} \frac{\Delta f}{f}+\frac{\gamma^{2}}{\gamma^{2}-\gamma_{t}^{2}} \frac{\Delta B}{B}
$$

The first term in equation (5) is readily calculated. The results given in Table 2 reflect the application of this correction. The revolution frequency is very accurately known and $\eta$ is known to about $10 \%$. Of the measurements considered in this memo, the largest deviation of the measured revolution frequency from $f_{c}$ was $4 \mathrm{~Hz}$. The resulting correction from the first term in equation (5) is $\Delta p=4.5 \mathrm{MeV} / \mathrm{c}$. In this case, the frequency correction contributes approximately $0.45 \mathrm{MeV} / \mathrm{c}$ to the uncertainty in the beam momentum measurement, or $0.27 \mathrm{~mm}\left(\delta L_{\eta}\right)$ to the overall uncertainty in the length of the central orbit.

The second term in equation (5) is difficult to estimate. Accordingly, no attempt is made to apply a $\Delta B$ correction to the measurements considered in this memo. This term is treated as a source of error in the measurement of the central orbit beam momentum.

The integrated bend field of the Accumulator changes due to variations in the temperature of the dipole magnets. An increase in the temperature of a dipole magnet causes two effects: (1) the length of the magnet expands (i.e. the path length of a particle in the magnet is increased), and (2) the gap between the pole faces increases - decreasing the dipole magnetic field. These two

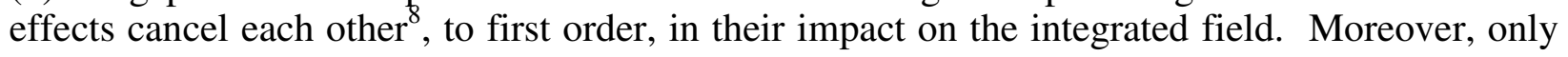
the second effect - the change in dipole field - is measured by the NMR readout of the reference dipole. Therefore, the net effect on the integrated dipole field is unknown and the second term in equation (5) cannot be accurately evaluated. A rough estimate of the importance of this term can be made by calculating its contribution using the NMR readout for the measurement with the greatest deviation from the nominal field. The largest deviation from $B_{o}$ in the results presented in Table 2 is 5.2 Gauss. If this were the actual $\Delta B$ in equation (5), the resulting correction to $\Delta p$ from the second term would be $0.52 \mathrm{MeV} / \mathrm{c}$. This corresponds to an orbit length error, $\delta L_{B}$, of $0.31 \mathrm{~mm}$.

\section{Overall Uncertainty in the Orbit Length}

The overall uncertainty in the orbit length measurement, $\delta L$, is a superposition of all of the uncertainties described above.

$$
\delta L=\delta L_{r e f} \oplus \delta L_{2} \oplus \delta L_{B P M} \oplus \delta L_{\eta} \oplus \delta L_{B}
$$


Adding in quadrature the estimates given above for each of these uncertainties yields the following estimates for $\delta L$ :

$$
\delta L \lesssim \begin{cases}1.6 \mathrm{~mm} & \text { Post-Lattice Upgrade } \\ 2.5 \mathrm{~mm} & \text { Pre-Lattice Upgrade }\end{cases}
$$

These are very conservative estimates of the uncertainty in the measurement of the Accumulator orbit length. From equation (3) the corresponding uncertainties in the determination of the beam momentum, $\delta p$, are:

$$
\delta p \lesssim\left\{\begin{array}{lll}
2.7 & \frac{\mathrm{MeV}}{\mathrm{c}} & \text { Post-Lattice Upgrade } \\
4.1 & \frac{\mathrm{MeV}}{\mathrm{c}} & \text { Pre-Lattice Upgrade }
\end{array}\right.
$$

\section{Beam Momentum and on the Accumulator Central Orbit}

The data taken as input for this analysis consist of closed orbit and revolution frequency measurements made before and after the Accumulator lattice upgrade. Both pre and post upgrade data consist of three different closed orbit measurements made with beam near the central orbit of the Accumulator. For each of these measurements, all of the 48 horizontal BPMs were in good working order. Figure 3 shows the horizontal difference between the measured orbit and the $\psi^{\prime}$ reference orbit for post-upgrade orbit measurements. Figure 4 shows the difference orbits for the post-upgrade measurements. The resulting beam momenta and orbit lengths derived from these orbits are summarized in Table 2 below. In Table 2 the subscript " $c$ " denotes a central orbit quantity and the subscript " $e$ " denotes an extraction orbit quantity.

\begin{tabular}{|c|c|c|c|c|c|c|c|}
\hline $\begin{array}{l}\text { Measure- } \\
\text { ment Date }\end{array}$ & $f_{r e v} \underline{10}(\mathrm{~Hz})$ & $\begin{array}{c}L_{c} \\
(\mathbf{m})\end{array}$ & $\begin{array}{c}P_{c} \\
(\mathrm{MeV} / \mathrm{c})\end{array}$ & $\begin{array}{c}L_{e} \\
(\mathbf{m})\end{array}$ & $\begin{array}{c}P_{e} \\
(\mathrm{MeV} / \mathrm{c})\end{array}$ & $\begin{array}{c}\Delta p \\
(M e V / c)\end{array}$ & $\begin{array}{c}\Delta L \\
(\mathrm{~mm})\end{array}$ \\
\hline $8 / 25 / 99^{1114}$ & 628837.00 & 474.0509 & 8799.60 & 474.1577 & 8882.40 & 82.80 & 106.8 \\
\hline $3 / 12 / 00$ & 628842.87 & 474.0552 & 8806.75 & 474.1619 & 8889.63 & 82.88 & 106.7 \\
\hline $6 / 22 / 00$ & 628840.06 & 474.0535 & 8803.89 & 474.1602 & 8886.74 & 82.85 & 106.7 \\
\hline \multicolumn{2}{|c|}{ Post-Upgrade Average: } & 474.0532 & 8803.41 & 474.1599 & 8886.26 & 82.85 & 106.7 \\
\hline $8 / 19 / 97$ & 628867.00 & 474.0455 & 8823.20 & 474.1938 & 8904.53 & 81.33 & 148.3 \\
\hline $9 / 24 / 96$ & 628868.50 & 474.0450 & 8822.25 & 474.1932 & 8903.57 & 81.32 & 148.2 \\
\hline $3 / 26 / 90$ & 628866.88 & 474.0395 & 8813.12 & 474.1878 & 8894.36 & 81.24 & 148.3 \\
\hline \multicolumn{2}{|c|}{ Pre-Upgrade Average: } & 474.0433 & 8819.52 & 474.1916 & 8900.82 & 81.30 & 148.3 \\
\hline
\end{tabular}

Table 29? Beam Energy Measurements before and after the Accumulator Lattice Upgrade

Figure 3 shows that all of the post-lattice upgrade orbits lie within $\pm 5 \mathrm{~mm}$ of the reference orbit and within $\pm 5 \mathrm{~mm}$ of each other. This is in marked contrast to the pre-upgrade orbits, which can deviate from the reference orbit by as much as $25 \mathrm{~mm}$.

The central orbit beam momenta $\left(p_{c}\right)$ reported in Table 2 reflect the application of a correction to account for the difference between the measured revolution frequency $\left(f_{\text {rev }}\right)$ and the revolution frequency associated with the canonical central orbit $\left(f_{c}\right)$. This correction is calculated from the first term in equation (5).

$$
\frac{d p}{p}=-\frac{1}{\eta} \frac{d f}{f}
$$

The value of $\eta$ is determined from synchrotron frequency measurements. The measurement of $\eta$ for the post-upgrade Accumulator lattice is presented in Appendix 2. 


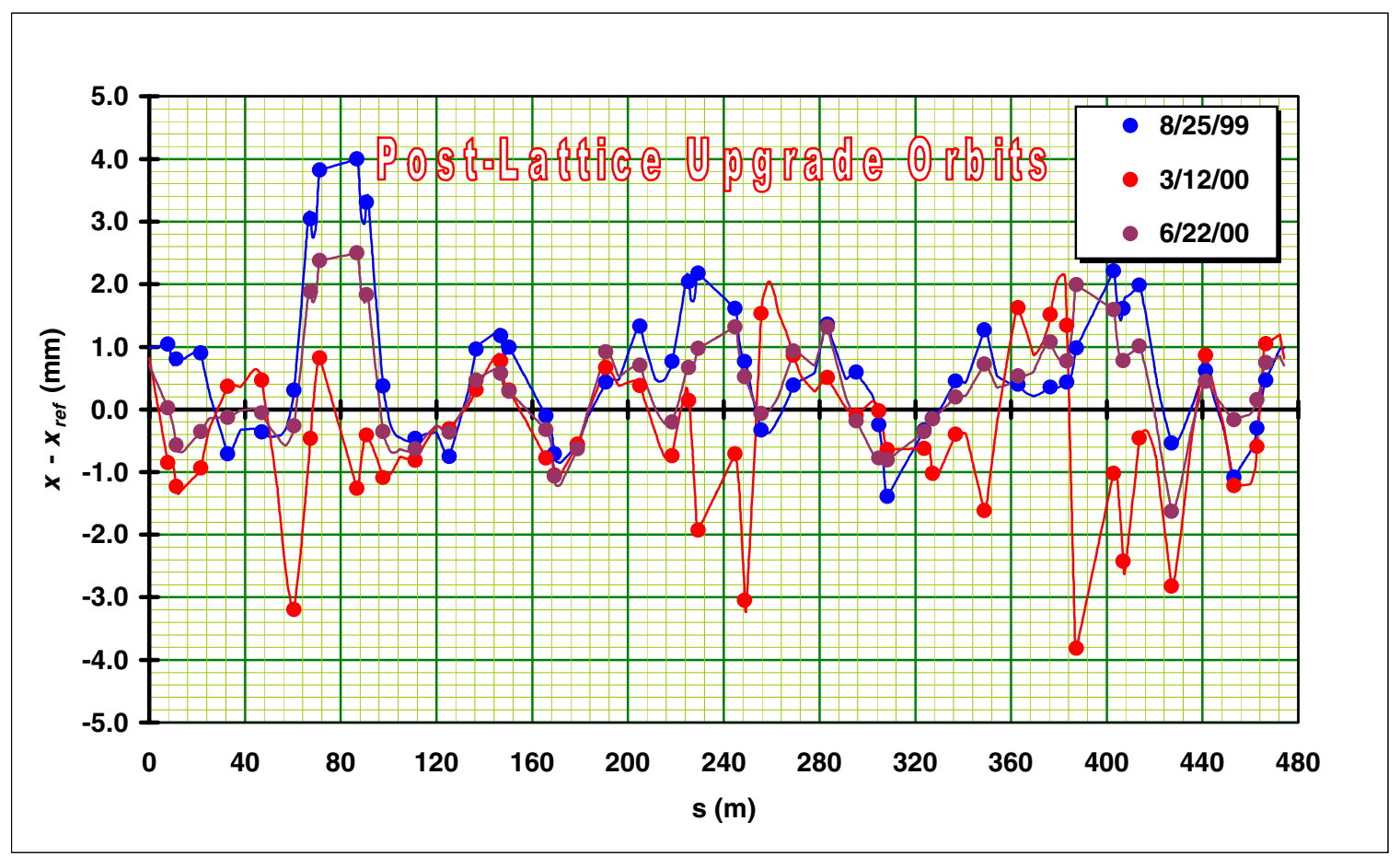

Figure 3 This figure shows the orbits used for the post-lattice upgrade beam energy measurements. The vertical axis measures the difference between the horizontal orbit $(x)$ and the reference orbit $\left(x_{r e f}\right)$ derived from the E835 $\psi^{\prime}$ scan of June 14, 2000. The solid continuous lines represent a model of the horizontal orbit parameterized by dipole kicks at all of the Accumulator dipoles and quadrupoles.

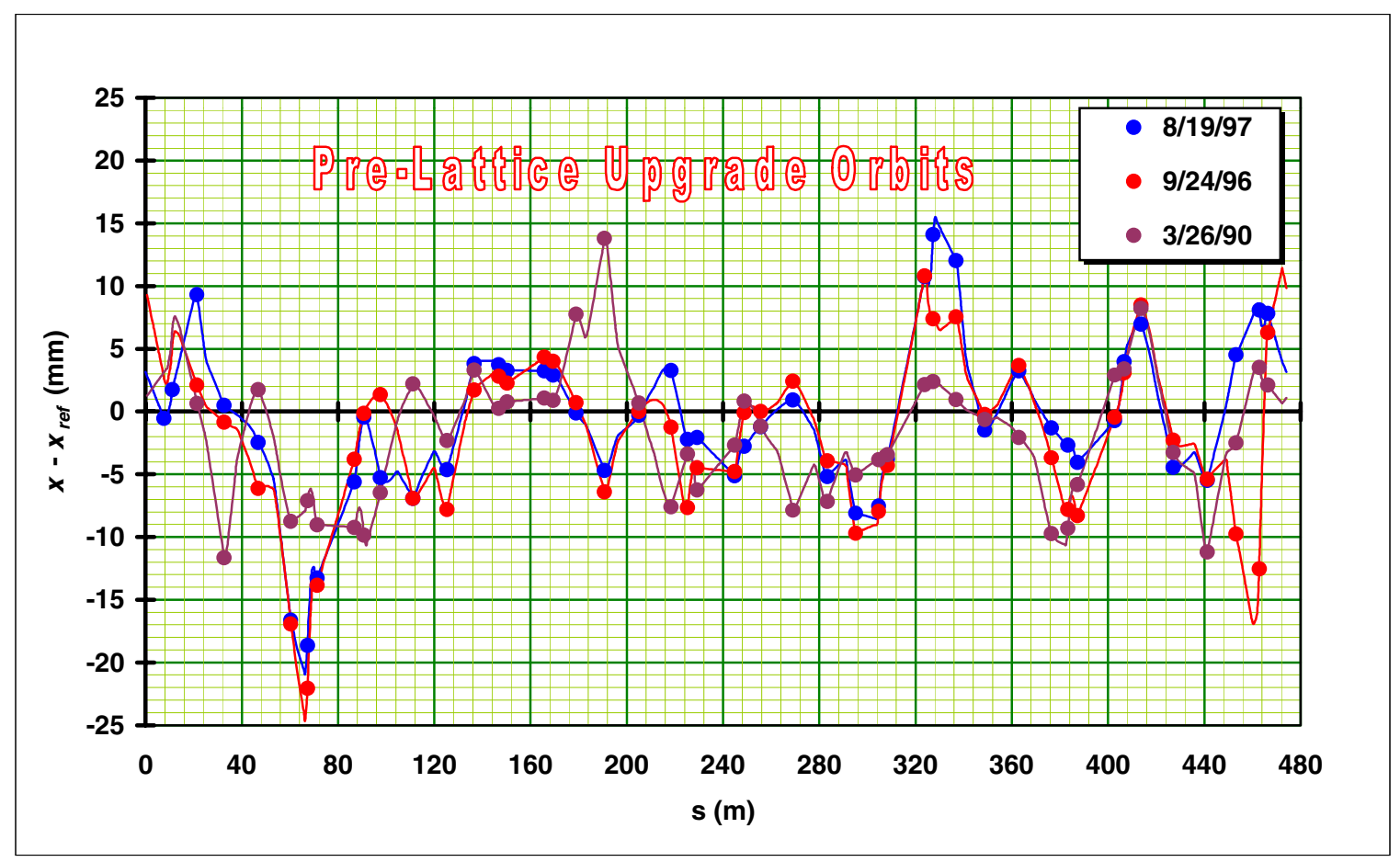

Figure 4 This figure shows the orbits used for the pre-lattice upgrade beam energy measurements. The vertical axis measures the difference between the horizontal orbit $(x)$ and the reference orbit $\left(x_{r e f}\right)$ derived from the E835 $\psi^{\prime}$ scan of June 14, 2000. The solid continuous lines represent a model of the horizontal orbit parameterized by dipole kicks at all of the Accumulator dipoles and quadrupoles. 
The central orbit lengths $\left(L_{c}\right)$ in Table 2 are calculated from the corrected central orbit momenta according to equation (10),

$$
L=\frac{c \beta}{f_{\text {rev }}}=\frac{1}{f_{\text {rev }}} \frac{p c}{\sqrt{(p c)^{2}+\left(m_{p} c^{2}\right)^{2}}}
$$

The average central orbit momenta and orbit lengths are given by:

$$
\begin{array}{cl}
\text { Post Lattice Upgrade: } & p_{c}=8803.4 \pm 2.7 \mathrm{MeV} / \mathrm{c} \\
& L_{c}=474.0532 \pm 0.0016 \mathrm{~m} \\
\text { Pre Lattice Upgrade: } & p_{c}=8819.5 \pm 4.1 \mathrm{MeV} / \mathrm{c} \\
& L_{c}=474.0433 \pm 0.0025 \mathrm{~m}
\end{array}
$$

\section{Beam Momentum and on the Accumulator Extraction Orbit}

The extraction orbit is defined, for purposes of this report, as the orbit taken by beam having a specific revolution frequency, hereinafter designated $f_{e}$, when the reference dipole field is $B_{o}$. In practice, this frequency is chosen so that beam extracted from the Debuncher circulates at the correct radial position in the Accumulator arcs. The values of $f_{e}$ for the pre-upgrade and postupgrade Accumulator are given in Table 1.

\section{Post-Lattice Upgrade Extraction Orbit Momentum}

The beam momentum and orbit length on the Accumulator extraction orbit can be calculated by integrating equation (9)] This is somewhat complicated by the fact that, on the upgrade lattice, $\gamma_{t}$, and hence $\eta$, varies significantly across the momentum aperture of the Accumulator. Moreover, the variation of $\eta$ with momentum is not well measured. The only information available at the present time is a single measurement of $\eta$ on the central orbit and one measurement on the extraction orbit. These measurements are described in Appendix 2 of this report. From this scant data, one can integrate equation (9) by assuming that $\eta$ varies linearly with frequency between the central and extraction orbits. Thus, $\eta(f)=\eta_{\mathrm{c}}+\alpha\left(f-f_{c}\right)$. The constant $\alpha$ is determined from the measurements of $\eta$ on the central and extraction orbits. With this form of $\eta(f)$, the integration of equation (9) proceeds as follows:

$$
\begin{aligned}
\ln \left(\frac{p_{e}}{p_{c}}\right) & =-\int_{f_{c}}^{f_{e}} \frac{d f}{\eta(f) f} \\
& =-\int_{f_{c}}^{f_{e}} \frac{d f}{f\left[\eta_{c}+\alpha\left(f-f_{c}\right)\right]} \\
& =-\frac{1}{\eta_{c}-\alpha f_{c}}\left[\int_{f_{c}}^{f_{e}} \frac{d f}{f}-\alpha \int_{f_{c}}^{f_{e}} \frac{d f}{\eta_{c}+\alpha\left(f-f_{c}\right)}\right] \\
& =-\frac{1}{\eta_{c}-\alpha f_{c}}\left\{\ln \left(\frac{f_{e}}{f_{c}}\right)-\ln \left[1-\frac{\alpha}{\eta_{c}}\left(f_{e}-f_{c}\right)\right]\right\} \\
p_{e} & =p_{c}\left(\frac{\eta_{e}}{\eta_{c}} \frac{f_{c}}{f_{e}}\right)^{\frac{1}{\eta_{c}-\alpha f_{c}}}
\end{aligned}
$$


where the subscript " $e$ " denotes extraction orbit quantities. Once the extraction orbit momentum is determined, the extraction orbit length is calculated from equation (10) The post-upgrade extraction orbit momentum and length results are tabulated in Table 2.

The uncertainty in $p_{e}$ is greatly affected by the uncertainty in the value of $\eta$. The value of $p_{e}$ is affected by variations in $p_{c}, \eta_{\mathrm{c}}$, and $\eta_{\mathrm{e}}$ according to:

$$
\begin{aligned}
\delta p_{e}=\frac{\partial p_{e}}{\partial p_{c}} \delta p_{c}+\frac{\partial p_{e}}{\partial \eta_{c}} \delta \eta_{c}+\frac{\partial p_{e}}{\partial \eta_{e}} \delta \eta_{e} \\
\frac{\delta p_{e}}{p_{e}}=\frac{\delta p_{c}}{p_{c}}-\frac{1}{\eta_{c}-\alpha f_{c}}\left[1+\frac{\eta_{c}}{\eta_{c}-\alpha f_{c}}\left(\frac{f_{e}}{f_{e}-f_{c}}\right) \ln \left(\frac{\eta_{e}}{\eta_{c}} \frac{f_{c}}{f_{e}}\right)\right] \frac{\delta \eta_{c}}{\eta_{c}} \\
+\frac{1}{\eta_{c}-\alpha f_{c}}\left[1+\frac{\eta_{e}}{\eta_{c}-\alpha f_{c}}\left(\frac{f_{c}}{f_{e}-f_{c}}\right) \ln \left(\frac{\eta_{e}}{\eta_{c}} \frac{f_{c}}{f_{e}}\right)\right] \frac{\delta \eta_{e}}{\eta_{e}}
\end{aligned}
$$

Using $\delta p_{c}=2.7 \mathrm{MeV} / \mathrm{c}$ from equation (8), and $\delta \eta_{\mathrm{c}} / \eta_{\mathrm{c}}=\delta \eta_{\mathrm{e}} / \eta_{\mathrm{e}}=10 \%$, and adding the contribution from the three terms on the right hand side of (12) in quadrature gives $\delta p_{e}=6.1 \mathrm{MeV} / \mathrm{c}$.

The uncertainty in the extraction orbit length is calculated by differentiating equation (10). The extraction orbit uncertainty is then given by:

$$
\delta L_{e}=\frac{L_{e}}{\gamma_{e}^{2}} \frac{\delta p_{e}}{p_{e}}
$$

For the post-upgrade measurements, $\delta L_{e}=3.6 \mathrm{~mm}$.

\section{Pre-Lattice Upgrade Extraction Orbit Momentum}

There are no recent measurements of the variation of $\eta$ with momentum for the pre-upgrade lattice. The Tevatron 1 Design Report 12 and the pre-upgrade Accumulator lattice model show $\eta$ to be very nearly constant between the central and extraction orbits. For constant $\eta$, the integration of equation (9) is straightforward. The resulting expression for the extraction orbit momentum is:

$$
p_{e}=p_{c}\left(\frac{f_{c}}{f_{e}}\right)^{\frac{1}{\eta}}
$$

The value of $\eta$ for the pre-upgrade Accumulator lattice has been measured to be: $\eta_{\mathrm{c}}=0.023$. The pre-upgrade extraction orbit momenta and lengths, calculated according to equations (14) and (10), are given in Table 2

The uncertainty in the in the extraction orbit momentum for the constant $\eta$ case is given by:

$$
\begin{aligned}
\delta p_{e} & =\frac{\partial p_{e}}{\partial p_{c}} \delta p_{c}+\frac{\partial p_{e}}{\partial \eta} \delta \eta \\
& =\frac{p_{e}}{p_{c}} \delta p_{c}-\ln \left(\frac{f_{c}}{f_{e}}\right) \frac{\delta \eta}{\eta^{2}}
\end{aligned}
$$


Using $\delta p_{c}=4.1 \mathrm{MeV} / \mathrm{c}$ from equation (8), and $\delta \eta / \eta=10 \%$, and adding the terms of the right hand side of (15) in quadrature one obtains: $\delta p_{e}=9.2 \mathrm{MeV} / \mathrm{c}$. The corresponding uncertainty in the length of the extraction orbit from equation (13) is $\delta L_{e}=5.4 \mathrm{~mm}$.

\section{Summary of Extraction orbit measurements}

The average extraction orbit momenta and orbit lengths are given by:

$$
\begin{array}{ll}
\text { Post Lattice Upgrade: } & p_{e}=8886.3 \pm 6.1 \mathrm{MeV} / \mathrm{c} \\
& L_{e}=474.1599 \pm 0.0036 \mathrm{~m} \\
\text { Pre Lattice Upgrade: } & p_{e}=8900.8 \pm 9.2 \mathrm{MeV} / \mathrm{c} \\
& L_{e}=474.1916 \pm 0.0054 \mathrm{~m}
\end{array}
$$

\section{Conclusions}

In December of 1999 it was discovered that the central orbit energy of the Debuncher was $57 \mathrm{MeV} / \mathrm{c}$ greater than the momentum corresponding to the center of the bunch rotation RF bucket ${ }^{13}$. Since the Debuncher bend bus had been adjusted to accept reverse proton beam from the Accumulator extraction channel, it was concluded that the Accumulator lattice upgrade had somehow effected a downward shift in the operating energy of the Antiproton Source.

The analysis of this memo suggests that the change in Accumulator beam energy is not large enough to account for what was observed in the Debuncher. The measurements analyzed here show a decrease in the extraction orbit momentum of only $14.6 \mathrm{MeV} / \mathrm{c}$. The momentum and orbit length differences from the pre-upgrade to the post-upgrade Accumulator lattice are summarized in Table 3 .

Table 3: Changes in Orbit Length and Momentum from the Accumulator Lattice Upgrade

\begin{tabular}{|l|c|c|c|}
\hline \multicolumn{1}{|c|}{ Orbit } & $\Delta f(\mathbf{H z})$ & $\Delta \boldsymbol{L}(\mathbf{m m})$ & $\Delta p(\mathrm{MeV} / \mathbf{c})$ \\
\hline \hline Central Orbit & -26.0 & 9.9 & -16.1 \\
\hline Extraction Orbit & 30.7 & -31.7 & -14.6 \\
\hline
\end{tabular}

These changes are consistent with equation (3) 


\section{References and Notes}

${ }^{1}$ A detailed description of the beam energy measurement is given in Pbar Note 633:

S.J. Werkema, Precision Measurement of the Accumulator Beam Energy, February 28, 2000. This document can be downloaded from the following URL:

http://cosmo.fnal.gov/documents/pbarnotes/pdf_files/PB633.PDF

${ }^{2}$ The June 14, $2000 \psi^{\prime}$ scan is comprised of E835 runs 5818 through 5837.

3 " $8 \mathrm{GeV}$ " as it's used here, refers to the approximate kinetic energy of the beam. In the parlance of the Fermilab Beams division, the term " $8 \mathrm{GeV}$ " refers to the stacking energy of the Accumulator (or the flattop energy of the Booster). The numerical value $8 \mathrm{GeV}$ is not used in any analysis nor is it the product of any of the calculations or measurements described in this memo.

${ }^{4} 1 \mathrm{mrad}$ is roughly half of the largest kick that can be delivered to the beam with dipole elements installed in the Accumulator. The Accumulator trim dipoles are capable of a 1.7 mrad kick at maximum excitation; the $25 \mathrm{Amp}$ shunt on a $5^{\circ}$ main bending dipole causes a $1.86 \mathrm{mrad}$ kick at maximum current.

${ }^{5}$ The test in reference 1 applies the orbit length calculation to the $\psi^{\prime}$ reference orbit of the 1997 fixed target run (pre-lattice upgrade) using the $\psi^{\prime}$ reference orbit from the June 14, 2000 (post-lattice upgrade) measurement of the $\psi^{\prime}$. The resulting orbit length was $0.27 \mathrm{~mm}$ shorter than the length that had been originally measured for this orbit.

${ }^{6}$ The evidence referred to here is an analysis of the BPM data from the E835 scan of the $\psi^{\prime}$ on June 14, 2000 given in Pbar Note 638. Most of the beam energy changes in this scan were conducted at constant bend field in the Accumulator. Under these circumstances, the only appreciable change in the orbit occurs in the high dispersion BPMs. Thus, the scaling of only the high dispersion BPMs are checked in this analysis. In addition, the effect that is being ascribed to BPM scaling errors could be the result of uncertainties in the Accumulator lattice parameters used in the analysis (primarily the dispersion at the horizontal BPMs). In that case, the scale errors of the high dispersion BPMs is less than what is indicated in Pbar Note 638. Pbar Note 638 can be downloaded from the following URL: http://cosmo.fnal.gov/documents/pbarnotes/pdf_files/PB638.PDF

${ }^{7}$ The reference dipole is a standard Accumulator $5^{\circ}$ dipole that is located external to the ring and powered from the Accumulator bend bus. An NMR field probe measures the magnetic field of the reference dipole. The NMR probe is read out to the accelerator control system via ACNET device A:BFIELD.

${ }^{8}$ There is a small residual change in the integrated field because the thermal expansion of the magnets is not isotropic.

${ }^{9}$ Explanation of Table 2:

$f_{\text {rev }}=$ Measured revolution frequency of the beam

$L_{c}=$ Measured length of the central orbit

$L_{e}=$ Length of the extraction orbit, calculated from equation (10)

$p_{c}=$ Measured beam momentum on the central orbit

$p_{e}=$ Beam momentum on the extraction orbit, calculated from equation (11) or (14)

$\Delta p=p_{e}-p_{c}$

$\Delta L=L_{e}-L_{c}$

${ }^{10} f_{\text {rev }}$ gives the revolution frequency at which the measurement was made. It should not be confused with the central orbit revolution frequency $\left(f_{c}\right)$. The values of orbit length and momentum in Table 2 reflect a correction to account for the momentum difference between $f_{\text {rev }}$ and $f_{c}$.

${ }^{11}$ Accumulator closed orbit file "Archive 9," measure on August 25, 1999, is the canonical post-lattice upgrade central orbit. This orbit measurement was made after the efforts to optimize the aperture and center the beam in the quadrupoles were complete.

${ }^{12}$ Design Report Tevatron 1 Project, September 1984 (page 5-4).

${ }^{13}$ D. McGinnis, Debuncher Energy Mismatch. Pbar Note 629, January 25, 2000. This Pbar Note can be downloaded from the following URL:

http://cosmo.fnal.gov/documents/pbarnotes/pdf_files/PB629.PDF 


\section{Appendix 1 - Reference Orbit BPM data}

June 14, $2000 \psi^{\prime}$ Reference Orbit BPM Data

E8335 Run 5827

$L_{r e f}=474.05428 \mathrm{~m}$

\begin{tabular}{|c|c|c|c|c|c|c|c|c|c|c|c|}
\hline \multicolumn{2}{|c|}{ Sector 1} & \multicolumn{2}{|c|}{ Sector 2} & \multicolumn{2}{|c|}{ Sector 3} & \multicolumn{2}{|c|}{ Sector 4} & \multicolumn{2}{|c|}{ Sector 5} & \multicolumn{2}{|c|}{ Sector 6} \\
\hline BPM & $\mathbf{x}(\mathbf{m m})$ & BPM & $\mathbf{x}(\mathbf{m m})$ & BPM & $\mathbf{x}(\mathbf{m m})$ & BPM & $\mathbf{x}(\mathbf{m m})$ & BPM & $\mathbf{x}(\mathbf{m m})$ & BPM & $\mathbf{x}(\mathbf{m m})$ \\
\hline $\mathrm{A} 1 \mathrm{Q} 01$ & -6.40 & $\mathrm{~A} 2 \mathrm{Q} 01$ & 1.97 & A3001 & -0.34 & $\mathrm{~A} 4 \mathrm{Q} 01$ & 1.54 & A5Q01 & 0.60 & $A 6 Q 01$ & 0.96 \\
\hline $\mathrm{A} 1003$ & -2.67 & $\mathrm{~A} 2 \mathrm{Q} 03$ & 1.17 & $\mathrm{~A} 3003$ & -0.50 & $\mathrm{~A} 4 \mathrm{Q} 03$ & 3.60 & $\mathrm{~A} 5 \mathrm{Q} 03$ & 4.02 & $\mathrm{~A} 6 \mathrm{Q} 03$ & 2.55 \\
\hline $\mathrm{A} 1 \mathrm{Q} 04$ & 2.18 & $\mathrm{~A} 2 \mathrm{Q} 04$ & 3.92 & $\mathrm{~A} 3004$ & 1.36 & $\mathrm{~A} 4 \mathrm{Q} 04$ & -0.20 & $\mathrm{~A} 5 \mathrm{Q} 04$ & 4.47 & $\mathrm{~A} 6 \mathrm{Q} 04$ & 2.90 \\
\hline $\mathrm{A} 1006$ & -1.74 & $\mathrm{~A} 2 \mathrm{Q} 06$ & 2.38 & $\mathrm{~A} 3006$ & -0.56 & $\mathrm{~A} 4 \mathrm{Q} 06$ & 1.27 & $\mathrm{~A} 5 \mathrm{Q} 06$ & -4.26 & $\mathrm{~A} 6 \mathrm{Q} 06$ & 0.21 \\
\hline $\mathrm{A} 1 \mathrm{Q} 08$ & 7.48 & $\mathrm{~A} 2 \mathrm{Q} 08$ & 8.53 & $\mathrm{~A} 3008$ & 1.06 & $\mathrm{~A} 4 \mathrm{Q} 08$ & 3.32 & A5Q08 & 1.49 & A6Q0 8 & 2.36 \\
\hline A1Q10 & 1.75 & $\mathrm{~A} 2 \mathrm{Q} 10$ & 0.66 & A3Q10 & -10.30 & $\mathrm{~A} 4 \mathrm{Q} 10$ & -5.81 & A5Q10 & -4.52 & A6Q10 & -17.61 \\
\hline $\mathrm{A} 1 \mathrm{Q} 11$ & -3.50 & $\mathrm{~A} 2 \mathrm{Q} 11$ & 1.24 & $\mathrm{~A} 3 \mathrm{Q} 11$ & -2.40 & $\mathrm{~A} 4 \mathrm{Q} 11$ & 1.80 & A5Q11 & 9.52 & A6Q11 & 1.65 \\
\hline A1Q14 & 1.61 & $\mathrm{~A} 2 \mathrm{Q} 14$ & -6.64 & A3Q14 & -1.14 & $\mathrm{~A} 4 \mathrm{Q} 14$ & -3.52 & A5Q14 & -0.35 & A6Q14 & -10.30 \\
\hline
\end{tabular}




\section{Appendix 2 - Measurement of $\eta$}

The values of accelerator parameter $\eta$ used in this report are calculated from measurements of the synchrotron frequency of bunched beam. The RF system used is ARF3 (downstream cavity only). ARF3 operates at $1.26 \mathrm{MHz}(h=2)$. The synchrotron frequency $\left(f_{\text {synch }}\right)$ is measured at several RF voltages $\left(V_{\text {cav }}\right)$. Figure 5 shows how a typical synchrotron frequency measurement is accomplished. The value of $\eta$ is calculated from the slope of the graph of $f_{\text {synch }}{ }^{2}$ versus $V_{\text {cav }}$ according to equation (16)

$$
\eta=\frac{2 \pi L p}{e h f_{r e v}} \frac{d f_{s y n c h}^{2}}{d V_{c a v}}
$$

Figure 6 shows the $f_{\text {synch }}^{2}$ versus $V_{\text {cav }}$ graphs for $\eta$ measurements made on two different dates with beam on the central and extraction orbits. The $V_{\text {cav }}$ and $f_{\text {synch }}$ data for these measurements are given in Table A - 1 and Table A - 2.

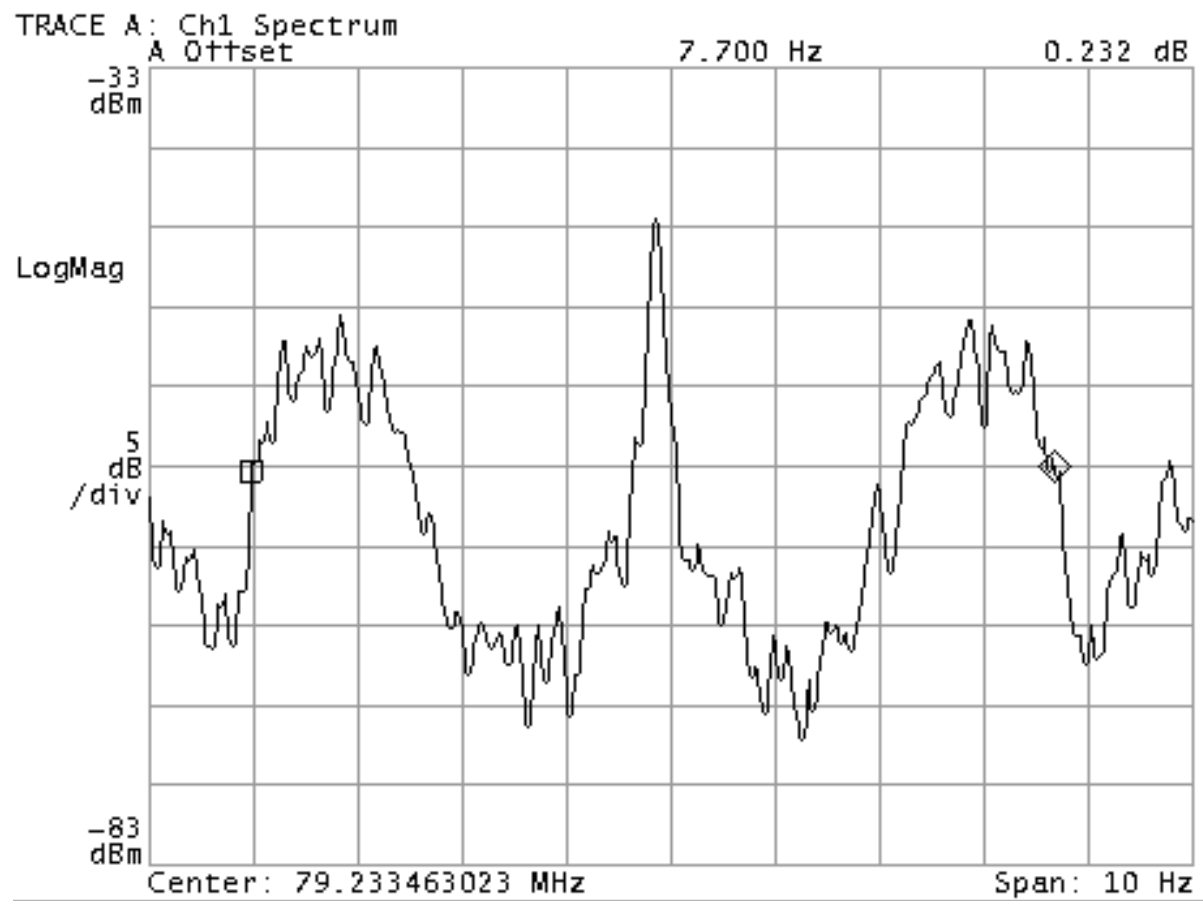

Figure 5 Measurement of $f_{\text {synch }}$. This figure shows the frequency spectrum of the Accumulator longitudinal Schottky pickup. The analyzer is tuned to $126 \times f_{\text {rev }}$. The beam was bunched to $V_{\text {cav }}=84.75$ Volts with the downstream cavity of ARF3. The frequency difference between the markers is $2 \times f_{\text {synch }}$.

\section{Central Orbit $\eta$ Measurement}

August 25, 1999. Pbar Electronic Logbook 1999 Entry 453

$\eta_{c}=0.01237$

$\gamma_{t}=6.508$

$f_{\text {rev }}=628836.9 \mathrm{~Hz}$ 
Table A - 1:

Central Orbit $f_{\text {synch }}$ vs. $V_{\text {cav }}$ Data

\begin{tabular}{|c|c|}
\hline $\begin{array}{c}\text { ARF3 Voltage } \\
\left(\boldsymbol{V}_{\text {cav }}\right) \\
(\text { Volts })\end{array}$ & $\begin{array}{c}\text { Synchrotron Freq. } \\
\left(\boldsymbol{f}_{\text {synch }}\right) \\
(\mathbf{H z})\end{array}$ \\
\hline \hline 84.75 & 3.8500 \\
\hline 155.50 & 5.3100 \\
\hline 387.50 & 8.2750 \\
\hline 597.50 & 10.0625 \\
\hline 785.00 & 11.9250 \\
\hline 1010.00 & 13.3500 \\
\hline 1277.50 & 15.1500 \\
\hline
\end{tabular}

\section{Extraction Orbit $\eta$ Measurement}

November 23, 1999. Pbar Electronic Logbook 1999 Entry 550

$\eta_{e}=0.01350$

$\gamma_{t}=6.385$

$f_{\text {rev }}=628765.5 \mathrm{~Hz}$

Table A - 2:

Extraction Orbit $f_{\text {synch }}$ vs. $V_{\text {cav }}$ Data

\begin{tabular}{|c|c|}
\hline $\begin{array}{c}\text { ARF3 Voltage } \\
\left(\boldsymbol{V}_{\text {cav }}\right) \\
(\text { Volts })\end{array}$ & $\begin{array}{c}\text { Synchrotron Freq. } \\
\left(\boldsymbol{f}_{\text {synch }}\right) \\
(\mathbf{H z})\end{array}$ \\
\hline \hline 166.50 & 5.4938 \\
\hline 537.50 & 9.9688 \\
\hline 825.50 & 12.7125 \\
\hline
\end{tabular}

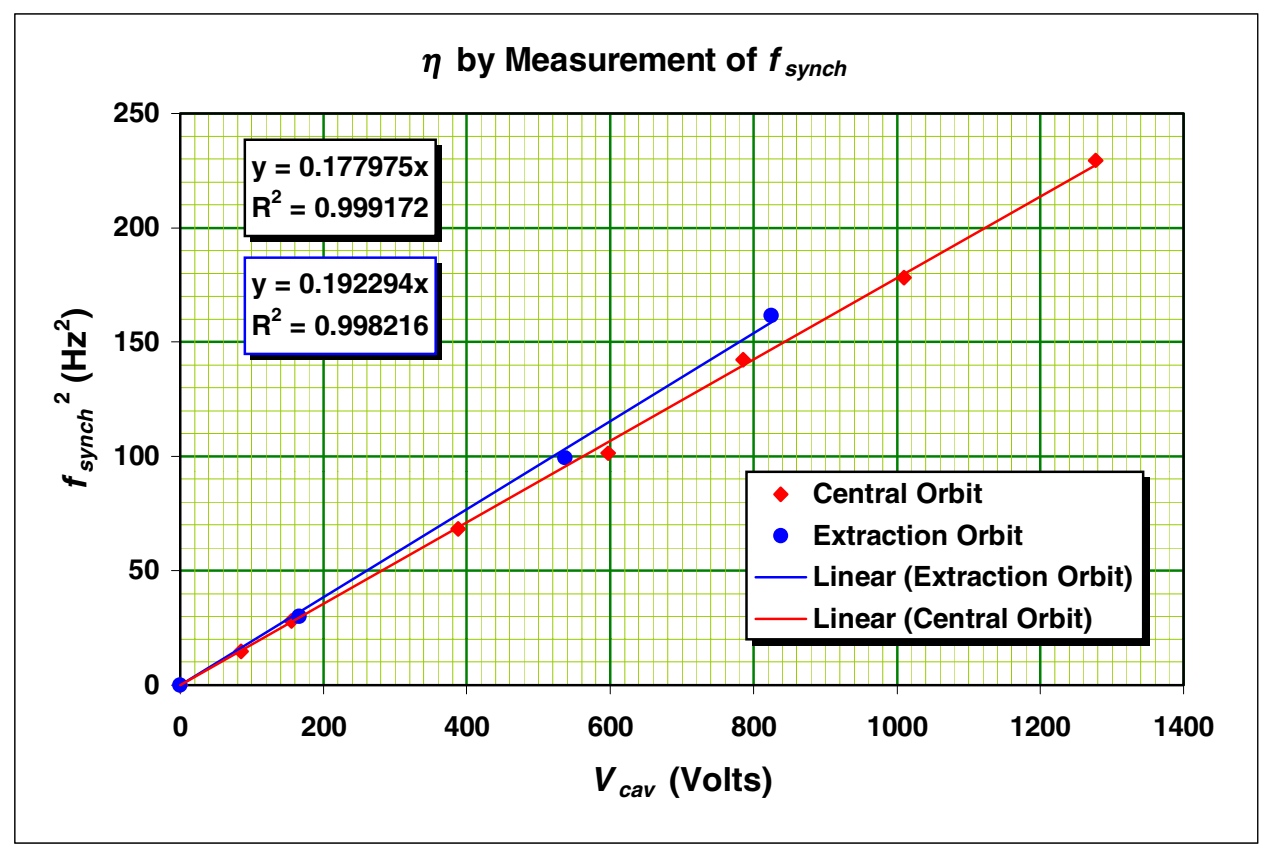

Figure 6 Plot of $f_{\text {synch }}{ }^{2}$ vs. $V_{\text {cav }}$ for the central and extraction orbit $\eta$ measurements. The value of $\eta$ is proportional to the slope of these graphs. 